\title{
Gravitational splitting at first order: Quantum information localization in gravity
}

\author{
William Donnelly* \\ Department of Physics, University of California, Santa Barbara, California 93106, USA \\ and Perimeter Institute for Theoretical Physics, 31 Caroline Street North, \\ Waterloo, Ontario N2L 2 Y5, Canada \\ Steven B. Giddings ${ }^{\dagger}$ \\ Department of Physics, University of California, Santa Barbara, California 93106, USA
}

(Received 10 July 2018; published 8 October 2018)

\begin{abstract}
We explore the important fundamental question of how quantum information is localized in quantum gravity, in a perturbative approach. Familiar descriptions of localization of information, such as via tensor factorization of the Hilbert space or a net of commuting subalgebras of operators, conflict with basic gravitational properties - specifically gauge invariance-already at leading order in perturbation theory. However, previous work found that information can be classically localized in a region in a way such that measurements, including those of the gravitational field, outside the region are insensitive to that information and only measure total Poincaré charges. This paper shows that, working to leading order in the gravitational coupling, a similar quantum result holds, leading to a definition of a "gravitational splitting" on the Hilbert space for gravity. Such localization of information also argues against a role for "soft hair" in resolving the information problem for black holes. This basic mathematical structure plausibly plays a foundational role in the quantum description of gravity.
\end{abstract}

DOI: 10.1103/PhysRevD.98.086006

\section{INTRODUCTION}

A long-standing question playing an important role in various approaches to quantum gravity is that of how quantum information is localized. For example, the idea that information can be equivalently represented in a dual "boundary" theory is central to the conjectured AdS/CFT equivalence, and the idea that information is accessible through the gravitational field has been suggested [1-3] as a possible resolution to the information problem for black holes. This question of localization of information in a gravitational theory arises because the usual notion of localization from local quantum field theory (LQFT) does not respect the gauge symmetries of gravity, which at least in the geometrical approximation correspond to diffeomorphisms. Put simply: how do we construct "qubits" in gravitational theories, and are they in any sense localized?

\footnotetext{
*Present address: Perimeter Institute for Theoretical Physics, 31 Caroline Street North, Waterloo, Ontario N2L 2Y5, Canada. wdonnelly@perimeterinstitute.ca † giddings@ucsb.edu

Published by the American Physical Society under the terms of the Creative Commons Attribution 4.0 International license. Further distribution of this work must maintain attribution to the author(s) and the published article's title, journal citation, and DOI. Funded by SCOAP ${ }^{3}$.
}

Specifically, local operators of LQFT are not gauge (diffeomorphism) invariant. There are various approaches to constructing operators that are gauge invariant. One involves constructing integrals over all of spacetime of products of local operators and is in a generic state maximally nonlocal; this type of construction is reviewed, e.g., in [4]. Another approach is to start with a local operator of LQFT and "gravitationally dress" it to make it gauge invariant. Explicit such constructions appear in [5], and related earlier constructions are those of $[6,7]$. These operators create not only particles, but also their gravitational fields, reflecting the statement that a particle is inseparable from its gravitational field. In particular, these operators must have nontrivial support extending to infinity, as shown in [8], so are also nonlocal, although if one works perturbatively in the gravitational coupling $\kappa=\sqrt{32 \pi G}$ the nonlocality is small in $\kappa$. But this raises the important question regarding whether in gravitational theory there is any precise notion of localized quantum information, ${ }^{1}$ or whether any information is necessarily delocalized in the collective state of a matter distribution and its gravitational field.

In fact, this question appears likely to play a fundamental role in construction of a theory of quantum gravity. This is

\footnotetext{
${ }^{1}$ For related discussion see [9].
} 
because the notion of a "subsystem," localizing information, is usually part of the fundamental structure assumed in a quantum theory. In finite systems, or locally finite ones such as a lattice, this localization arises from a tensor factorization of the Hilbert space. In LQFT, such localization is also a fundamental starting principle [10], encoded in the statement that subalgebras of operators localized to spacelike separated regions commute. It is both interesting and apparently important that these statements appear to be significantly modified in gravity. Plausibly a good approach towards understanding quantum gravity is to take a "quantum-first" viewpoint that it should respect basic axioms of quantum mechanics, particularly the existence of a Hilbert space; then, a large part of the problem of formulating the theory is to find appropriate mathematical structure on that Hilbert space for describing gravity $[11,12]$. This is guided partly by a correspondence principle for weak gravitational fields. In such an approach, it seems that a basic element of the mathematical structure is plausibly such a notion of subsystems, just as this notion enters at the foundational level for LQFT and other quantum systems.

A key point appears to be that while one is required to nonlocally gravitationally dress local operators, there is significant latitude in how an operator is dressed. This led to description in [13] of how one could begin with a classical matter configuration in a given region and find a corresponding classical gravitational field such that gravitational (or other) observations outside that region could not detect the details of the configuration. This (and a related construction in QED) suggested the idea of a "gravitational splitting," space of states that is indistinguishable by measurements outside a region. This paper will focus on the question of construction of such gravitational splittings in the quantum theory of matter plus gravity, working to leading order in $\kappa$. Construction of such splittings demonstrates a way to localize quantum information at this order. It also appears to lend weight to arguments [13] that soft hair does not offer a way to determine the information content of a region, which plausibly extend to the case of black hole interiors.

\section{THE QUESTION OF LOCALIZING QUANTUM INFORMATION}

The present goal is to understand the extent to which information can be "localized" in a gravitational theory, at least perturbatively. We first recall how information is localized in nongravitational local quantum field theory (LQFT).

In finite or locally finite (such as a lattice) quantum systems, information is localized by providing a tensor factorization of the Hilbert space, e.g.,

\footnotetext{
${ }^{2}$ This was called split structure in [13].
}

$$
\mathcal{H}=\bigotimes_{i} \mathcal{H}_{i}
$$

where $\mathcal{H}_{i}$ are a collection of Hilbert spaces. Then, states can be independently excited in the different tensor factors of the Hilbert space. However, the Hilbert space of LQFT does not have such a structure, due to the generic von Neumann type-III behavior of its operator algebras. ${ }^{3}$ This means that if we for example divide space into two at a surface, the vacuum $|0\rangle$ of LQFT has infinite entanglement between excitations on the two sides of the division, and simple product states are in a different Hilbert space than $|0\rangle$.

However, in LQFT we can describe localized information through the existence of subalgebras of operators associated with different regions. If $U_{1}$ and $U_{2}$ are two spacelike separated regions, then operators compactly supported in these different regions form commuting subalgebras. These operators can be thought of as creating independent excitations in the two regions. In general, the collection, or net, of such subalgebras mirrors the open set structure (topology) of the spacetime manifold [10], and effectively defines a subsystem structure.

Indeed, one can go a step closer to (1), and make it clear that independent information can exist "in a region" in LQFT. This arises from the notion of a split vacuum. Let $U$ be a neighborhood, and $U_{\epsilon}$ be an $\epsilon$-extended open set containing $U$. Denote the subalgebra associated to $U$ as $\mathcal{A}_{U}$, and that associated to the complement $U_{\epsilon}^{\prime}$ as $\mathcal{A}_{U_{\epsilon}^{\prime}}$. Then, it has been shown (see [10] and references therein) that there exists a split vacuum $\left|U_{\epsilon}\right\rangle$ so that for any $A \in \mathcal{A}_{U}$ and $A^{\prime} \in U_{\epsilon}^{\prime}$,

$$
\left\langle U_{\epsilon}\left|A A^{\prime}\right| U_{\epsilon}\right\rangle=\langle 0|A| 0\rangle\left\langle 0\left|A^{\prime}\right| 0\right\rangle:
$$

observations outside $U_{\epsilon}$ are incapable of distinguishing operation of different operators inside $U$. So, e.g., we could pick a collection of operators $A_{I} \in \mathcal{A}_{U}$ and form the states

$$
\left|\psi_{I}\right\rangle=A_{I}\left|U_{\epsilon}\right\rangle
$$

observations using operators $A^{\prime} \in \mathcal{A}_{U_{c}^{\prime}}$ cannot distinguish such states, and so these states can be thought of as describing localized information-if $I=1,2$, a localized qubit.

We next turn to the analogous question for a gravitational theory, taken for simplicity to be that of a mass- $m$ scalar $\phi$ minimally coupled to gravity, with Lagrangian

$$
\mathcal{L}=\frac{2}{\kappa^{2}} R-\frac{1}{2}\left[(\nabla \phi)^{2}+m^{2} \phi^{2}\right] .
$$

We work perturbatively about flat space, although these considerations generalize; e.g., the AdS context is described in [16]. The essential problem is that $\phi(x)$ is no longer an observable: it is not gauge invariant under

\footnotetext{
${ }^{3}$ See [14], and other references in [15].
} 
diffeomorphisms, which act infinitesimally with parameters $\xi^{\mu}(x)$ as $\delta x^{\mu}=\kappa \xi^{\mu}$ and

$$
\delta_{\xi} \phi(x)=-\kappa \xi^{\mu} \partial_{\mu} \phi(x) .
$$

Working perturbatively in $\kappa$, [5] showed that one may "dress" $\phi$ to give gauge-invariant operators $\Phi(x)$, but these must [8] involve integrals to infinity of the metric perturbation $h_{\mu \nu}(x)$, defined by

$$
g_{\mu \nu}=\eta_{\mu \nu}+\kappa h_{\mu \nu}
$$

and thus obey a nonlocal algebra [5,17].

In essence, as noted, an operator that creates a particle must also create its gravitational field. This complicates the question of localization of information in gravity, since one then expects that information about the particle state is contained in the gravitational field and thus may be measured far from the particle. But the question is how much information is contained in the gravitational field, and specifically whether there is localized information that is not measurable via the gravitational field outside a region.

If gravity permits global symmetries, there is in fact a trivial example of localization of information. Suppose that $\phi_{1}(x)$ and $\phi_{2}(x)$ are two fields related by such a global symmetry, and consider corresponding identically dressed operators $\Phi_{1}(x)$ and $\Phi_{2}(x)$. Then, there is no way to asymptotically perturbatively distinguish ${ }^{4}$ states created by $\Phi_{1}(x)$ or $\Phi_{2}(x)$ : these operators can be used to create "localized gravitational qubits," analogous to (3). However, the existence of such global symmetries in a gravitational theory has long been questioned, either because of black hole evaporation, spacetime wormholes [18], or the nature of symmetries in string theory [19]. While the ultimate role of global symmetries in quantum gravity is unclear, we seek to examine the more general question of localization of information, without such a symmetry.

\section{GRAVITATIONAL DRESSING OF OPERATORS AND STATES}

We first need to revisit and extend the discussion of gravitational dressing given in [5]. There, it was explicitly found that to leading order in $\kappa$, the LQFT operator $\phi(x)$ could be promoted to a diffeomorphism-invariant operator $\Phi(x)$. This was done by finding a dressing $V^{\mu}(x)$ linear in $h_{\mu \nu}$ and transforming as

$$
\delta_{\xi} V^{\mu}(x)=\kappa \xi^{\mu}(x)
$$

under an infinitesimal diffeomorphism, which to leading order in $\kappa$ acts as

\footnotetext{
${ }^{4}$ For some discussion of the nonperturbative situation, see [13].
}

$$
\delta_{\xi} h_{\mu \nu}=-\partial_{\mu} \xi_{\nu}-\partial_{\nu} \xi_{\mu}
$$

Then,

$$
\Phi(x)=\phi\left(x^{\mu}+V^{\mu}(x)\right)
$$

is diffeomorphism invariant to $\mathcal{O}(\kappa)$. The dressing $V^{\mu}(x)$ is not unique; different choices exist, including the gravitational line and Coulomb dressings investigated in [5].

To promote a more general LQFT operator $A$ to a diffeomorphism-invariant version $\hat{A}$, note that the diffeomorphisms are generated by the constraints

$$
\mathcal{C}_{\mu}=\left(\kappa T_{\mu \nu}-\frac{4}{\kappa} G_{\mu \nu}\right) n^{\nu}
$$

with $T_{\mu \nu}$ the stress tensor, $G_{\mu \nu}$ the Einstein tensor, and $n^{\mu}=$ $(1,0,0,0)$ the unit timelike vector. This may e.g., be seen from the covariant canonical approach [20-26], as reviewed in $[8,16]$. So, a diffeomorphism-invariant observable $\hat{A}$ should commute with $\mathcal{C}_{\mu}$ :

$$
\left[\mathcal{C}_{\mu}(x), \hat{A}\right]=0 .
$$

The approach taken will be to solve the condition (11) perturbatively in an expansion in $\kappa$, starting with the $\kappa=0$ operator $A$ of LQFT. To begin, the expansion of the metric (6) gives

$$
G_{\mu \nu}=\kappa G_{\mu \nu}^{(1)}-\frac{\kappa^{2}}{4} t_{\mu \nu}
$$

where $G_{\mu \nu}^{(1)}$ is linear in $h$, and $t_{\mu \nu}$ is an effective stress tensor that is quadratic and higher order in $h$. Then the constraints (10) become

$$
\mathcal{C}_{\mu}=c_{\mu}+\kappa\left(T_{\mu \nu}+t_{\mu \nu}\right) n^{\nu},
$$

where $c_{\mu}=-4 G_{\mu \nu}^{(1)} n^{\nu}$. Explicitly,

$$
\begin{aligned}
& c_{0}=-2\left(\partial_{i} \partial_{j} h_{i j}-\partial_{i} \partial_{i} h_{j j}\right), \\
& c_{i}=-2\left(\partial_{j} \dot{h}_{i j}-\partial_{i} \dot{h}_{j j}+\partial_{i} \partial_{j} h_{0 j}-\partial_{j} \partial_{j} h_{0 i}\right) .
\end{aligned}
$$

Equal-time commutators of $c_{\mu}$ with the $h_{\mu \nu}$ generate the linearized diffeomorphisms (8), and correspondingly, as seen in (7), a dressing $V^{\mu}(x)$ should satisfy

$$
\left[c_{\mu}(t, \vec{x}), V^{\nu}\left(t, \vec{x}^{\prime}\right)\right]=i \kappa \delta_{\mu}^{\nu} \delta^{3}\left(\vec{x}-\vec{x}^{\prime}\right) .
$$

This may be explicitly checked, e.g., using a covariant gauge fixing as in Appendix B of [5]. Then, given a dressing $V_{\mu}$ satisfying (7), (15), the invariance condition (11) is easily seen to be solved to $\mathcal{O}(\kappa)$ by 


$$
\begin{aligned}
\hat{A} & =A+i \int d^{3} x V^{\mu}(x)\left[T_{0 \mu}(x), A\right]+\mathcal{O}\left(\kappa^{2}\right) \\
& =e^{i \int d^{3} x V^{\mu}(x) T_{0 \mu}(x)} A e^{-i \int d^{3} x V^{\mu}(x) T_{0 \mu}(x)}+\mathcal{O}\left(\kappa^{2}\right)
\end{aligned}
$$

[generalizing Eq. (33) of [5]].

To understand whether states can carry local information, one also needs the dressed version of a state such as $\left|\psi_{I}\right\rangle=A_{I}\left|U_{\epsilon}\right\rangle$. Equation (16) suggests

$$
\left|\hat{\psi}_{I}\right\rangle=\left[1+i \int d^{3} x V^{\mu}(x) T_{0 \mu}(x)\right]\left|\psi_{I}\right\rangle+\mathcal{O}\left(\kappa^{2}\right)
$$

as the analogous dressed state. Here it suffices to assume that the state is built on a vacuum $\left|U_{\epsilon}\right\rangle_{\phi}$ which is split just for $\phi$; for $h$ this is the usual vacuum. As in Gupta-Bleuler quantization of gauge theory, we do not require $\mathcal{C}_{\mu}(x)$ to annihilate dressed physical states [27], but only that it have vanishing matrix elements between such states:

$$
\left\langle\hat{\psi}_{I}\left|\mathcal{C}_{\mu}(x)\right| \hat{\psi}_{J}\right\rangle=0
$$

If one assumes that the positive-frequency part of $c_{\mu}$ annihilates the vacuum at $\mathcal{O}\left(\kappa^{0}\right)$,

$$
c_{\mu}^{(+)}(x)\left|U_{\epsilon}\right\rangle_{\phi}=0,
$$

then (18) follows to order $\kappa$ from the definition (17) and from (15).

We could alternately try to introduce a split vacuum for $h_{\mu \nu}$ and seek to enforce the vanishing of the constraints (18) via an expression similar to (17), involving $t_{\mu \nu}$. However, the gauge noninvariance of $t_{\mu \nu}$ is an added complication. While this would be useful if we wanted to consider states corresponding to dressed gravitons in $U$, this construction will not be needed here and will be deferred to future work.

\section{FIRST-ORDER GRAVITATIONAL SPLITTING}

Given the preceding constructions, and particularly the flexibility in choosing the dressing $V^{\mu}$, the next question is whether one can choose a dressing so that different excitations localized to a neighborhood $U$ can be dressed in a way so they are indistinguishable outside the extended neighborhood $U_{\epsilon}$. Of course, all such excited states $\left|\psi_{I}\right\rangle$ yield a nontrivial gravitational field outside $U_{\epsilon}$, so the question is in what circumstances this dressing is insensitive to the state.

Specifically, since we are working to $\mathcal{O}(\kappa)$, consider the matrix element

$$
\left\langle\hat{\psi}_{I}\left|h_{\mu \nu}(\bar{x})\right| \hat{\psi}_{J}\right\rangle
$$

between two states of the form (17). In a more complete treatment, we really want to use a diffeomorphisminvariant, dressed, version of $h_{\mu \nu}(\bar{x})$, but to leading order in $\kappa$, (20) suffices. Ultimately, we are also interested in higher-point functions, e.g., $\left\langle\hat{\psi}_{I}\left|h_{\mu \nu}(\bar{x}) h_{\lambda \sigma}(\bar{y})\right| \hat{\psi}_{J}\right\rangle$, etc., but these involve the $\mathcal{O}\left(\kappa^{2}\right)$ corrections that have been neglected. So, we will check insensitivity of (20) to the state only to first order in $\kappa$.

For $\bar{x} \in U_{\epsilon}^{\prime},\left\langle\psi_{I}\left|h_{\mu \nu}(\bar{x})\right| \psi_{J}\right\rangle=0$, and one finds

$$
\begin{aligned}
& \left\langle\hat{\psi}_{I}\left|h_{\mu \nu}(\bar{x})\right| \hat{\psi}_{J}\right\rangle \\
& =-i\left\langle\psi_{I}\left|\int d^{3} x T_{0 \lambda}(x)\left[V^{\lambda}(x), h_{\mu \nu}(\bar{x})\right]\right| \psi_{J}\right\rangle+\mathcal{O}\left(\kappa^{2}\right) \\
& =-i \int d^{3} x\left[V^{\lambda}(x), h_{\mu \nu}(\bar{x})\right]\left\langle\psi_{I}\left|T_{0 \lambda}(x)\right| \psi_{J}\right\rangle+\mathcal{O}\left(\kappa^{2}\right),
\end{aligned}
$$

where the last line uses the linearity of $V$ in $h$, so that $[V, h]$ is a c-number.

The next goal is to find a dressing $V^{\mu}(x)$ that is "as insensitive as possible" to the details of the matter distribution inside $U$. This is done by first picking a point $y \in U$. Then, we construct the dressing by "dressing the point $y$ " with some "standard" dressing $V_{S}^{\mu}(y)$ and then adding dressing connecting the point $y$ to a more general point $x \in U_{\epsilon}$. Specifically, consider the expression [12]

$$
\begin{aligned}
V_{L \mu}(x, y)= & -\frac{\kappa}{2} \int_{y}^{x} d x^{\prime \nu}\left\{h_{\mu \nu}\left(x^{\prime}\right)-\int_{y}^{x^{\prime}} d x^{\prime \prime \lambda}\left[\partial_{\mu} h_{\nu \lambda}\left(x^{\prime \prime}\right)\right.\right. \\
& \left.\left.-\partial_{\nu} h_{\mu \lambda}\left(x^{\prime \prime}\right)\right]\right\}
\end{aligned}
$$

Given the transformation (8), one may easily check that

$$
\begin{aligned}
\delta_{\xi} V_{L \mu}(x, y)= & \kappa\left\{\xi_{\mu}(x)-\xi_{\mu}(y)\right. \\
& \left.+\frac{1}{2}\left(x^{\nu}-y^{\nu}\right)\left[\partial_{\mu} \xi_{\nu}(y)-\partial_{\nu} \xi_{\mu}(y)\right]\right\} .
\end{aligned}
$$

Next, pick a particular standard dressing $V_{S}^{\mu}(y)$, satisfying (7); this could, e.g., be either the gravitational line or Coulomb dressings of [5]. Then, combining (7) and (23) shows that

$V^{\mu}(x)=V_{L}^{\mu}(x, y)+V_{S}^{\mu}(y)+\frac{1}{2}(x-y)_{\nu}\left[\partial^{\nu} V_{S}^{\mu}(y)-\partial^{\mu} V_{S}^{\nu}(y)\right]$

satisfies the correct transformation law (7).

The dressing (24) is just what is needed for maximum insensitivity to the details of the state. To see this, note that by the split property (2), the integrand of (21) vanishes for $x$ outside $U_{\epsilon}$, and this means the term involving $V_{L}$ does not contribute. As a result, one finds

$$
\begin{aligned}
\left\langle\hat{\psi}_{I}\left|h_{\mu \nu}(\bar{x})\right| \hat{\psi}_{J}\right\rangle= & \tilde{h}_{\mu \nu}^{S \lambda}(\bar{x}, y)\left\langle\psi_{I}\left|P_{\lambda}\right| \psi_{J}\right\rangle \\
& +\frac{1}{2} \partial_{y}^{\lambda} \tilde{h}_{\mu \nu}^{S \sigma}(\bar{x}, y)\left\langle\psi_{I}\left|M_{\lambda \sigma}\right| \psi_{J}\right\rangle+\mathcal{O}\left(\kappa^{2}\right),
\end{aligned}
$$


where we have defined a collection of standard dressing fields, labeled by $\lambda$,

$$
\tilde{h}_{\mu \nu}^{S \lambda}(\bar{x}, y)=-i\left[h_{\mu \nu}(\bar{x}), V_{S}^{\lambda}(y)\right],
$$

and where $P_{\mu}$ and $M_{\mu \nu}$ are the total momenta and angular momenta operators,

$$
\begin{aligned}
P_{\mu} & =-\int d^{3} x T_{0 \mu}(x), \\
M_{\mu \nu} & =-\int d^{3} x\left[(x-y)_{\mu} T_{0 \nu}(x)-(x-y)_{\nu} T_{0 \mu}(x)\right] .
\end{aligned}
$$

In short, the matrix elements of the metric outside $U_{\epsilon}$ only depend on the matrix elements of the Poincaré charges. Of course no localized state can be an eigenstate of all the Poincare charges, but any subspace of states where these matrix elements take a given fixed value produces identical matrix elements for $h_{\mu \nu}$ outside the neighborhood.

Thus a subspace of such states localized to $U_{\epsilon}$ can encode information not accessible by $\mathcal{O}(\kappa)$ measurements of the gravitational field $h_{\mu \nu}$ outside. A gravitational splitting is defined as $[13]^{5}$ a collection of Hilbert subspaces $\mathcal{H}_{U_{\epsilon}}^{i} \subset \mathcal{H}$ so that for any two states $|\psi\rangle,\left|\psi^{\prime}\right\rangle \in \mathcal{H}_{U_{\epsilon}}^{i}$, and any operator $\bar{A}$ localized outside $U_{\epsilon}$,

$$
\left\langle\psi|\bar{A}| \psi^{\prime}\right\rangle=\left\langle\psi \mid \psi^{\prime}\right\rangle\langle i|\bar{A}| i\rangle,
$$

so the value of $\bar{A}$ is independent of the choice of state within $\mathcal{H}_{U_{e}}^{i}$. The preceding construction thus yields a gravitational splitting to leading order $\kappa$, with the labels $i$ given by the values of the Poincare charges in the individual subspaces. This generalizes the classical result of [13] and implies the existence of gravitational dressing such that the quantum information contained in a matter configuration, aside from its Poincaré charges, can be localized in a neighborhood, to this order. An important question is generalizing this construction to higher order.

Given a nongravitational LQFT state localized to a neighborhood, the preceding construction gives a dressing of it that, outside the enlarged neighborhood, creates initial data for a gravitational field determined by the Poincaré charges and the choice of standard field. This can be thought of as arising from adding a radiation field (solution of the source-free equations) to a given gravitational field configuration to put it in standard form. As noted, examples are a gravitational line dressing field generalizing that found in [5], or the generalization of the Schwarzschild dressing field found in [5], giving in general a linearized boosted Kerr field. The existence of such field configurations connects to results for the full nonlinear theory in the literature. In particular, [28] (also see [29]) showed that

\footnotetext{
${ }^{5}$ In [13] this was called a "split structure."
}

initial data for the fully nonlinear gravitational field arising from a localized matter distribution may be restricted to a cone going to infinity, generalizing the gravitational line, and [30] showed that the initial data may be chosen to be boosted Kerr outside a neighborhood, albeit in the special case of a solution of the vacuum equations. Of course, in general as these fields evolve forward, they will produce outgoing radiation. For example, the gravitational line initial data produce radiation as it settles down to a Coulomb configuration.

The preceding results also appear pertinent to the question of whether soft hair $[2,3]$ can help with the problem of unitarizing black hole evolution. Specifically, given a region $U$, these results show that initial data may be constructed for the gravitational field such that outside $U_{\epsilon}$, the field only depends on the Poincare charges of a matter distribution inside $U$. The values of the soft charges will depend on which standard dressing is chosen outside $U_{\epsilon}$, but not on the other details of the state inside $U$. Moreover, other values of the soft charges may be found by superposing a homogeneous (sourceless) solution of the linearized gravitational field equations on whatever standard dressing has been chosen. ${ }^{6}$ So, while there is a large amount of extra information in the soft charges, it is uncorrelated with the information in $U$; the former information parameterizes the additional gravitational radiation that has been added on top of the underlying configuration. ${ }^{7}$ While the preceding analysis has been carried out in a flat background, one certainly expects that if there is such "localized" information that is invisible to soft charges outside a general region, there is likewise localized information inside a black hole. Work is in progress to further describe properties of dressing in black hole backgrounds [33].

The preceding discussion also suggests a diminished role for higher multipoles of the gravitational field in the AdS/ CFT correspondence. It has been argued that, since all multipoles fall at the same rate in AdS, that could play an important role in a holographic correspondence [34,35]. However, similar dressing constructions have been given in AdS [16] and plausibly can be used to argue that higher multipoles can be likewise removed by choice of radiation field.

Finally, as noted in the introduction, gravitational splittings or related concepts may play an important role in the foundational structure of a theory of quantum gravity. Starting with Hilbert space, one needs to find a mathematical structure on it that reproduces spacetime structure in the weak-gravity correspondence limit. One approach to specification of such a "gravitational substrate" [11] is plausibly in terms of a network of Hilbert spaces, such as arising from gravitational splittings, and related by inclusion.

\footnotetext{
${ }^{6}$ The basic soft-charge analysis is given in terms of linearized perturbations, so higher-order effects are not considered.

${ }^{7}$ For related arguments, see $[31,32]$.
} 


\section{ACKNOWLEDGMENTS}

The work of S. B. G. was supported in part by the U.S. Department of Energy under Contract No. DE-SC0011702, and that of W. D. was supported in part by the University of California and in part by Perimeter Institute for Theoretical
Physics. Research at Perimeter Institute is supported by the Government of Canada through Innovation, Science and Economic Development Canada and by the Province of Ontario through the Ministry of Research, Innovation and Science.
[1] S. W. Hawking, The Information Paradox for Black Holes, arXiv:1509.01147.

[2] S. W. Hawking, M. J. Perry, and A. Strominger, Soft Hair on Black Holes, Phys. Rev. Lett. 116, 231301 (2016); arXiv:1601.00921.

[3] S. W. Hawking, M. J. Perry, and A. Strominger, Superrotation charge and supertranslation hair on black holes, J. High Energy Phys. 05 (2017) 161; arXiv:1611.09175.

[4] S. B. Giddings, D. Marolf, and J. B. Hartle, Observables in effective gravity, Phys. Rev. D 74, 064018 (2006); arXiv: hep-th/0512200.

[5] W. Donnelly and S. B. Giddings, Diffeomorphism-invariant observables and their nonlocal algebra, Phys. Rev. D 93, 024030 (2016); arXiv:1507.07921.Erratum, Phys. Rev. D94, 029903(E) (2016).

[6] I. Heemskerk, Construction of bulk fields with gauge redundancy, J. High Energy Phys. 09 (2012) 106; arXiv: 1201.3666.

[7] D. Kabat and G. Lifschytz, Decoding the hologram: Scalar fields interacting with gravity, Phys. Rev. D 89, 066010 (2014); arXiv:1311.3020.

[8] W. Donnelly and S. B. Giddings, Observables, gravitational dressing, and obstructions to locality and subsystems, Phys. Rev. D 94, 104038 (2016); arXiv:1607.01025.

[9] T. Jacobson, Boundary unitarity and the black hole information paradox, Int. J. Mod. Phys. D 22, 1342002 (2013); arXiv:1212.6944.

[10] R. Haag, Local Quantum Physics: Fields, Particles, Algebras, Texts and Monographs in Physics (Springer, Berlin, 1992).

[11] S. B. Giddings, Quantum-first gravity, arXiv:1803.04973.

[12] S. B. Giddings, Quantum gravity: A quantum-first approach, arXiv:1805.06900.

[13] W. Donnelly and S. B. Giddings, How is quantum information localized in gravity?, Phys. Rev. D 96, 086013 (2017); arXiv:1706.03104.

[14] D. Buchholz and R. Verch, Scaling algebras and renormalization group in algebraic quantum field theory, Rev. Math. Phys. 07, 1195 (1995); arXiv:hep-th/9501063.

[15] J. Yngvason, The role of type III factors in quantum field theory, Rep. Math. Phys. 55, 135 (2005); arXiv: math-ph/0411058.

[16] S. B. Giddings and A. Kinsella, Gauge-invariant observables, gravitational dressings, and holography in AdS, arXiv:1802.01602.

[17] S. B. Giddings, Hilbert space structure in quantum gravity: An algebraic perspective, J. High Energy Phys. 12 (2015) 099; arXiv:1503.08207.
[18] S. B. Giddings and A. Strominger, Axion induced topology change in quantum gravity and string theory, Nucl. Phys. B306, 890 (1988).

[19] T. Banks and L. J. Dixon, Constraints on string vacua with space-time supersymmetry, Nucl. Phys. B307, 93 (1988).

[20] A. Ashtekar and A. Magnon-Ashtekar, On the symplectic structure of general relativity, Commun. Math. Phys. 86, 55 (1982).

[21] A. Ashtekar, L. Bombelli, and R. Koul, Phase space formulation of general relativity without a $3+1$ splitting, Lect. Notes Phys. 278, 356 (1987).

[22] C. Crnkovic and E. Witten, in Three Hundred Years of Gravitation, edited by S. Hawking and W. Israel (Cambridge University Press, Cambridge, England, 1986).

[23] C. Crnkovic, Symplectic geometry of the covariant phase space, Classical Quantum Gravity 5, 1557 (1988).

[24] G. J. Zuckerman, in Mathematical Aspects of String Theory: Proceedings, edited by S. Yau (World Scientific, Singapore, 1987), pp. 259-284.

[25] J. Lee and R. M. Wald, Local symmetries and constraints, J. Math. Phys. (N.Y.) 31, 725 (1990).

[26] V. Iyer and R. M. Wald, Some properties of Noether charge and a proposal for dynamical black hole entropy, Phys. Rev. D 50, 846 (1994); arXiv:gr-qc/9403028.

[27] S. N. Gupta, Quantization of Einstein's gravitational field: Linear approximation, Proc. Phys. Soc. 65, 161 (1952).

[28] A. Carlotto and R. Schoen, Localizing solutions of the Einstein constraint equations, Inventiones Mathematicae 205, 559 (2016).

[29] P. T. Chruściel, Anti-gravity à la Carlotto-Schoen, arXiv: 1611.01808 .

[30] J. Corvino and R. M. Schoen, On the asymptotics for the vacuum Einstein constraint equations, J. Diff. Geom. 73, 185 (2006); arXiv:gr-qc/0301071.

[31] M. Mirbabayi and M. Porrati, Dressed Hard States and Black Hole Soft Hair, Phys. Rev. Lett. 117, 211301 (2016); arXiv:1607.03120.

[32] R. Bousso and M. Porrati, Soft hair as a soft wig, Classical Quantum Gravity 34, 204001 (2017); arXiv:1706.00436.

[33] S. B. Giddings and S. Weinberg (to be published).

[34] G. T. Horowitz and V. E. Hubeny, CFT description of small objects in AdS, J. High Energy Phys. 10 (2000) 027; arXiv: hep-th/0009051.

[35] D. Marolf, W. Kelly, and S. Fischetti, in Springer Handbook of Spacetime, edited by A. Ashtekar and V. Petkov (Springer, New York, 2014), pp. 381-407; arXiv: 1211.6347. 